\title{
Study of changes in levels of salivary estriol and progesterone in preterm labor in comparison to normal pregnancy
}

\author{
Richa $\operatorname{Yadav}^{1 *}$, Urmila Singh ${ }^{2}$
}

\begin{abstract}
${ }^{1}$ Department of Obstetrics and Gynecology, Autonomous State Medical College, Bahraich, Uttar Pradesh, India ${ }^{2}$ Department of Obstetrics and Gynecology, KGMU, Lucknow, Uttar Pradesh, India
\end{abstract}

Received: 17 October 2019

Accepted: 13 November 2019

\author{
*Correspondence: \\ Dr. Richa Yadav, \\ E-mail: yadavricha965@gmail.com
}

Copyright: (C) the author(s), publisher and licensee Medip Academy. This is an open-access article distributed under the terms of the Creative Commons Attribution Non-Commercial License, which permits unrestricted non-commercial use, distribution, and reproduction in any medium, provided the original work is properly cited.

\begin{abstract}
Background: This prospective research was designed to evaluate changes in levels of salivary Estriol and Progesterone in preterm labor in comparison to normal pregnancy. Spontaneous onset of labor at term is produced by definite increase in estriol and fall in progesterone. This rise in free estrogen should precede the onset of labor according with the known effect of estrogen and progesterone on myometrial activity. Concentration of steroid in saliva reflect unbound unconjugated and biologically active fraction.

Methods: The present study was carried for duration of one year enrolling 115 antenatal women between 28 and $<37$ weeks of gestation with or without labour pains attending the antenatal clinic in KGMU, Lucknow and those admitted in Queen Mary's Hospital Lucknow. $5 \mathrm{ml}$ of saliva was collected in cryovials from each patient to estimate estriol and progesterone. The estimation of saliva progesterone and estriol was done by immunoenzymatic colorimetric method of the supernatant of sample.

Results: In our study mean value of saliva progesterone levels of pregnant women with preterm delivery (study group IIA) was lower than the control group (Group l) but the difference was not significant $(3814.46 \pm 751.14 \mathrm{pg} / \mathrm{ml} \mathrm{versus}$ $3945.16 \pm 577.11 \mathrm{pg} / \mathrm{ml}, \mathrm{p}=0.351)$. Mean value of estriol study group who delivered preterm $(3512.85 \pm 586.16 \mathrm{pg} / \mathrm{ml})$ was higher as compared to the mean value of control group $(2691.72 \pm 681.08 \mathrm{pg} / \mathrm{ml})$ and difference was significant (p value <0.001).

Conclusions: Thus, there is significant rise in level of saliva estriol, it can be used as a predictor for detecting symptomatic and asymptomatic women at risk for preterm birth.
\end{abstract}

Keywords: Estriol, Preterm labour, Progesterone, Saliva

\section{INTRODUCTION}

Preterm birth defined as any delivery at less than 37 completed weeks of gestation ( $<259$ days). The lower limit of viability being generally accepted to be at 23 completed weeks. Preterm birth is a heterogeneous condition; up to $30-40 \%$ of all cases of preterm birth are the results of elective delivery for a maternal or a fetal complication. The remaining $60-70 \%$ of preterm birth is probably the result of covert or sub-clinical infective/ inflammatory processes, cervical dysfunction, idiopathic (unknown causes), multiple gestations and possible social, nutritional and environment interaction. ${ }^{1}$ This report focuses on this latter group of so-called, spontaneous preterm births.

The diagnosis of preterm labour which precedes preterm delivery has been traditionally based on clinical indicators, including a history of preterm birth, symptoms and clinical examination. Progesterone is essential for maintenance of pregnancy and promote uterine relaxation 
indicators have been shown to have limited value in the prediction of spontaneous preterm delivery. ${ }^{2}$

Spontaneous onset of labour at term is produced by a definite increase in the saliva estriol to progesterone ratio. That a rise in free estrogen should precede the onset of labour according with the known effect of estrogen and progesterone on myometrial activity. ${ }^{3,4}$ Concentration of steroid in saliva reflects unbound, unconjugated and biologically active fraction. As saliva is easy to collect and store, measurement can be readily introduced. Saliva estriol levels showed a very high correlation $(r=0.98)$ with serum levels of free estriol in pregnant women and salivary estriol levels were suggested as a means for the assessment of fetoplacental function. ${ }^{5,6}$

Salivary progesterone levels showed good correlation $(\mathrm{r}=$ 0.47-0.58) with serum levels during menstrual cycle and reflected the serum progesterone levels. ${ }^{7,8}$ So this study was undertaken in the light to determine level of saliva estriol and progesterone in preterm labour and normal pregnancy.

\section{METHODS}

The study was a prospective study conducted in department of obstetrics and gynaecology, King George Medical University, Lucknow in collaboration with department of biochemistry, King George Medical University, Lucknow over a period of one year (July 2013 to July 2014).

\section{Inclusion criteria}

- Gestational age between 28- < 37 weeks having threatened preterm labour (uterine contractions with cervix $<80 \%$ effaced and $<1 \mathrm{~cm}$ dilated) or established preterm labour pains with according to guidelines of ACOG (1997).

\section{Exclusion criteria}

- Not willing to participate

- Patients who were taking steroids

- Who were having connective tissue disorder, gout or metabolic disorder like viral hepatitis

- Patients with fetal congenital anomalies, intrauterine death, fetal distress

- Patients with oral infection, poor oral hygiene or recent oral injuries.

\section{Sample collection}

After per abdominal and per vaginal examinations, verbal consent was taken. In women who came with preterm labour sample was collected before any intervention. Participants were instructed to rinse mouth with water to remove food residue before sample collection for 10 minutes.
A $5 \mathrm{ml}$ of saliva was collected in cryovials. Samples were taken to the department of biochemistry, KGMU, Lucknow where the samples were centrifuged at 3000 rpm for 15 minutes. The samples were maintained at $4{ }^{\circ} \mathrm{C}$ for no longer than 2 hours. If the specimens cannot assay within this time, the samples were stored at temperature of $-20^{\circ} \mathrm{C}$. The supernatant was further quantitatively tested for estriol and progesterone.

\section{Estimation of progesterone and estriol}

The estimation of saliva progesterone and estriol was done by immunoenzymatic colorimetric method manufactured by Diametra S.r.l. headquater: Garibaldi, 18-20090 SEGRATE (MI) Italy. Lowest detectable concentration of Progesterone was $3.27 \mathrm{pg} / \mathrm{ml}$ at the $95 \%$ confidence interval and that of estriol that can be distinguished was $1 \mathrm{pg} / \mathrm{ml}$ at the confidence limit $95 \%$.

Total number of 115 patients were enrolled and followed till delivery and they were divided into two Group as shone below in the pie Figure 1.

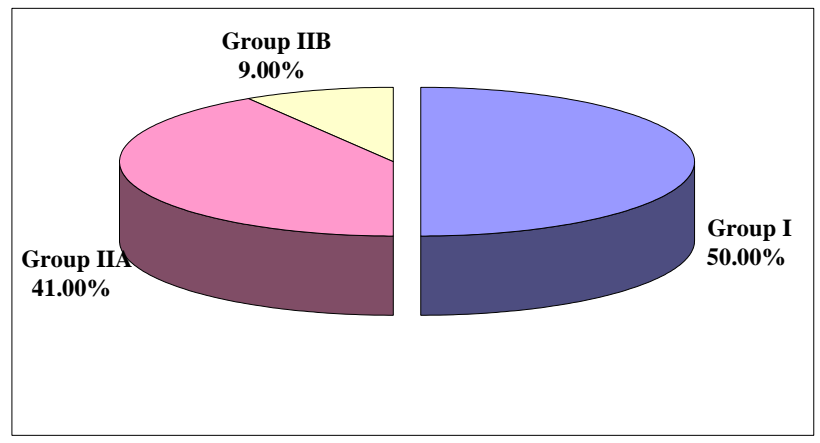

Figure 1: Distribution of total study group

Control group (Group I): Patient with normal pregnancy with no preterm labour pain. Total 60 patients were enrolled they were followed till delivery. Out of 60,8 were lost, 50 delivered at full term and two patients had preterm delivery they were excluded from study. So, in control group total 50 patients were included $(n=50)$.

Study group (Group II): Patients who came with preterm labour pains. Total 55 patients were enrolled, patient followed till delivery 5 patients were lost to follow $(\mathrm{n}=$ 50). The study group was further subdivided into two groups.

Study group, (IIA): Patient who came with preterm labour pains, tocolysis given but had preterm delivery (n $=41$ ). Study group (IIB): Patient who came with preterm labour pains, tocolysis given and patient delivered at full term $(\mathrm{n}=09)$.

\section{RESULTS}

Table 1 shows that majority of the study population $(52 \%)$ were aged below 25 years. Difference in age of the 
women in the above groups was not found to be significant $(\mathrm{p}=0.307)$. Between group comparison of Group I and Group II, revealed that proportion of women aged 26-30 years was found to be higher in Group I as compared to Group II while proportion of women aged 30-35 years was found to be higher in Group II as compared to Group I but this difference was not found to be statistically significant $(\mathrm{p}=0.163)$. Between group comparison of Group I and Group IIA, revealed that proportion of women aged up to 25 years and 26-30 years was found to be higher in Group I as compared to Group IIA while proportion of women aged 30-35 years was found to be higher in Group IIA as compared to Group I but this difference was not found to be statistically significant $(\mathrm{p}=0.146)$.

Table 1: Age profile of study population.

\begin{tabular}{|c|c|c|c|c|c|c|c|c|}
\hline \multirow{2}{*}{ Age (years) } & \multicolumn{2}{|c|}{ Group I $(\mathbf{n}=\mathbf{5 0})$} & \multicolumn{2}{|c|}{ Group IIA $(\mathrm{n}=41)$} & \multicolumn{2}{|c|}{ Group IIB $(n=9)$} & \multicolumn{2}{|c|}{ Group II $(\mathbf{n}=\mathbf{5 0})$} \\
\hline & No. & $\%$ & No. & $\%$ & No. & $\%$ & No. & $\%$ \\
\hline Up to 25 & 26 & 52.00 & 20 & 48.78 & 6 & 66.67 & 26 & 52.00 \\
\hline $26-30$ & 20 & 40.00 & 12 & 29.27 & 2 & 22.22 & 14 & 28.00 \\
\hline $30-35$ & 4 & 8.00 & 9 & 21.95 & 1 & 11.11 & 10 & 20.00 \\
\hline \multirow{5}{*}{\multicolumn{2}{|c|}{$\begin{array}{l}\text { Statistical significance } \\
\left(\chi^{2} \text { test }\right)\end{array}$}} & \multicolumn{3}{|c|}{ Group I, Group IIA and Group IIB } & \multicolumn{3}{|c|}{$\chi^{2}=4.811 ; p=0.307$} & \\
\hline & & \multicolumn{3}{|c|}{ Between Group I and Group II } & \multicolumn{3}{|c|}{$\chi^{2}=3.630 ; p=0.163$} & \\
\hline & & \multicolumn{3}{|c|}{ Between Group I and Group IIA } & \multicolumn{3}{|c|}{$\chi^{2}=3.853 ; ' p '=0.146$} & \\
\hline & & \multicolumn{3}{|c|}{ Between Group I and Group IIB } & \multicolumn{3}{|c|}{$\chi^{2}=1.036 ;{ }^{\prime} p '=0.596$} & \\
\hline & & \multicolumn{3}{|c|}{ Between Group IIA and Group IIB } & \multicolumn{3}{|c|}{$\chi^{2}=1.018 ; p=0.601$} & \\
\hline
\end{tabular}

Table 2: Gravida status of study population.

\begin{tabular}{|c|c|c|c|c|c|c|c|c|}
\hline \multirow{2}{*}{ Gravida } & \multicolumn{2}{|c|}{ Group I $(\mathbf{n}=\mathbf{5 0})$} & \multicolumn{2}{|c|}{ Group IIA $(n=41)$} & \multicolumn{2}{|c|}{ Group IIB (n = 9) } & \multicolumn{2}{|c|}{ Group II $(\mathbf{n}=\mathbf{5 0})$} \\
\hline & No. & $\%$ & No. & $\%$ & No. & $\%$ & No. & $\%$ \\
\hline 1 & 24 & 48.00 & 20 & 48.78 & 4 & 44.44 & 24 & 48.00 \\
\hline 2 & 15 & 30.00 & 7 & 17.07 & 1 & 11.11 & 8 & 16.00 \\
\hline 3 & 9 & 18.00 & 8 & 19.51 & 2 & 22.22 & 10 & 20.00 \\
\hline $4+$ & 2 & 4.00 & 6 & 14.63 & 2 & 22.22 & 8 & 16.00 \\
\hline \multirow{5}{*}{\multicolumn{2}{|c|}{$\begin{array}{l}\text { Statistical significance } \\
\left(\chi^{2} \text { test }\right)\end{array}$}} & \multirow{2}{*}{\multicolumn{3}{|c|}{$\begin{array}{l}\text { Group I, Group IIA and Group IIB } \\
\text { Between Group I and Group II }\end{array}$}} & \multicolumn{3}{|c|}{$\chi^{2}=6.379 ; p=0.382$} & \\
\hline & & & & & \multicolumn{3}{|c|}{$\chi^{2}=5.783 ; p=0.123$} & \\
\hline & & \multicolumn{3}{|c|}{ Between Group I and Group IIA } & \multicolumn{3}{|c|}{$\chi^{2}=4.485 ; p=0.214$} & \\
\hline & & \multicolumn{3}{|c|}{ Between Group I and Group IIB } & \multicolumn{3}{|c|}{$\chi^{2}=4.832 ; \mathrm{p}^{\prime}=0.184$} & \\
\hline & & \multicolumn{3}{|c|}{ Between Group IIA and Group IIB } & \multicolumn{3}{|c|}{$\chi^{2}=0.486 ; p=0.922$} & \\
\hline
\end{tabular}

Table 3: Period of gestation.

\begin{tabular}{|c|c|c|c|c|c|c|c|c|}
\hline \multirow{2}{*}{$\begin{array}{l}\text { Period of } \\
\text { gestation }\end{array}$} & \multicolumn{2}{|c|}{ Group I $(\mathbf{n}=\mathbf{5 0})$} & \multicolumn{2}{|c|}{ Group IIA $(n=41)$} & \multicolumn{2}{|c|}{ Group IIB $(\mathbf{n}=9)$} & \multicolumn{2}{|c|}{ Group II $(\mathbf{n}=\mathbf{5 0})$} \\
\hline & No. & $\%$ & No. & $\%$ & No. & $\%$ & No. & $\%$ \\
\hline$<32$ weeks & 16 & 32.00 & 5 & 12.20 & 7 & 77.78 & 12 & 24.00 \\
\hline $32-35$ weeks & 30 & 60.00 & 23 & 56.10 & 1 & 11.11 & 24 & 48.00 \\
\hline$>35$ weeks & 4 & 8.00 & 13 & 31.71 & 1 & 11.11 & 14 & 28.00 \\
\hline \multirow{5}{*}{\multicolumn{2}{|c|}{$\begin{array}{l}\text { Statistical significance } \\
\left(\chi^{2} \text { test }\right)\end{array}$}} & \multicolumn{3}{|c|}{ Group I, Group IIA and Group IIB } & \multicolumn{3}{|c|}{$\chi^{2}=22.635 ; p<0.001$} & \\
\hline & & \multicolumn{3}{|c|}{ Between Group I and Group II } & \multicolumn{3}{|c|}{$\chi^{2}=6.794 ; p=0.033$} & \\
\hline & & \multicolumn{3}{|c|}{ Between Group I and Group IIA } & \multicolumn{3}{|c|}{$\chi^{2}=10.665 ; p=0.005$} & \\
\hline & & \multicolumn{3}{|c|}{ Between Group I and Group IIB } & \multicolumn{3}{|c|}{$\chi^{2}=7.657 ; p=0.022$} & \\
\hline & & \multicolumn{3}{|c|}{ Between Group IIA and Group IIB } & \multicolumn{3}{|c|}{$\chi^{2}=17.455 ; p<0.001$} & \\
\hline
\end{tabular}

\section{Obstetric profile}

Between group comparison of Group I and II, Gravida 1 women were found in equal proportion in both the groups. Gravida 2 were found in higher proportion of Group I while gravid 3 and 4 were found in higher proportion in group IIB. But between the group comparison the difference was not significant (Table 2).

Period of gestation in majority of study population (54\%) was 32-35 weeks. On comparison of Group I and Group IIA, period of gestation < 32 weeks and 32-35 weeks was 
found in higher in Group I as compared to Group II and period of gestation > 35 weeks was found in higher proportion of Group IIA as compared to Group I and this difference was found to significant $(\mathrm{p}=0.005)$. Between Group comparison of Group I and Group IIB, period of gestation < 32 weeks were found in higher proportion in Group IIB and this difference was found to be significant $(\mathrm{p}=0.022)($ Table 3$)$.

Progesterone levels of Group I $(3945.16 \pm 577.11 \mathrm{pg} / \mathrm{ml})$ was found to be highest followed by that of Group IIA $(3814.46 \pm 751.14 \mathrm{pg} / \mathrm{ml})$ and lowest of Group IIB (3588.67 $\pm 1022.60 \mathrm{pg} / \mathrm{ml})$.
Intergroup comparison difference in progesterone levels of Group I, Group IIA and Group IIB was not found to be statistically significant $(\mathrm{p}=0.325)$.

Between group difference in progesterone levels of Group I and Group IIA too were not found to be statistically significant $(\mathrm{p}=0.351)$. Between group difference in progesterone levels of Group I and Group IIB was also not found to be statistically significant ( $\mathrm{p}=$ 0.449). Between group difference in progesterone levels of Group IIA and Group IIB was also not found to be statistically significant $(\mathrm{p}=0.449)($ Table 4$)$.

Table 4: Progesterone values of study group and control group.

\begin{tabular}{|llllll|}
\hline & $\begin{array}{l}\text { Number of } \\
\text { subjects }\end{array}$ & $\begin{array}{c}\text { Minimum prg } \\
\text { level }(\mathrm{pg} / \mathrm{ml})\end{array}$ & $\begin{array}{l}\text { Maximum Prg } \\
\text { level }(\mathrm{pg} / \mathrm{ml})\end{array}$ & $\begin{array}{c}\text { Mean prg level } \\
(\mathrm{pg} / \mathrm{ml})\end{array}$ & $\begin{array}{c}\text { Standard } \\
\text { deviation }\end{array}$ \\
\hline Group I & 50 & 2217.00 & 5656.00 & 3945.16 & 577.11 \\
\hline Group IIA & 41 & 2215.00 & 5129.00 & 3814.46 & 751.14 \\
\hline Group IIB & 9 & 1401.00 & 4722.00 & 3588.67 & 1022.60 \\
\hline \multirow{3}{*}{$\begin{array}{l}\text { Statistical } \\
\text { significance }\end{array}$} & Group I, Group IIA and Group IIB (ANOVA) & $\mathrm{F}=1.139 ; \mathrm{p}=0.324$ \\
\cline { 2 - 5 } & Between Group I and Group IIA (Student ' $\mathrm{t}$ ' test) & ' $\mathrm{t}$ ' $=0.938 ; \mathrm{p}=0.351$ \\
\cline { 2 - 5 } & Between Group I and Group IIB (Student ' $\mathrm{t}$ ' test) & ' $\mathrm{t}$ ' $=1.496 ; \mathrm{p}=0.140$ \\
\cline { 2 - 5 } & Between Group IIA and Group IIB (Student ' $\mathrm{t}$ ' test) & ' $\mathrm{t}$ ' $=0.764 ; \mathrm{p}=0.449$ \\
\hline
\end{tabular}

Table 5: Comparison of progesterone levels at different gestational age $(<32$ weeks).

\begin{tabular}{|c|c|c|c|c|c|}
\hline & $\begin{array}{l}\text { Number of } \\
\text { subjects }\end{array}$ & $\begin{array}{l}\text { Minimum prg } \\
\text { level (pg/ml) }\end{array}$ & $\begin{array}{l}\text { Maximum prg } \\
\text { level (pg/ml) }\end{array}$ & $\begin{array}{l}\text { Mean prg level } \\
(\mathrm{pg} / \mathrm{ml})\end{array}$ & $\begin{array}{l}\text { Standard } \\
\text { deviation }\end{array}$ \\
\hline \multicolumn{6}{|c|}{ At Gestational age $<32$ weeks } \\
\hline Group I & 16 & 3242.00 & 5449.00 & 3901.75 & 553.20 \\
\hline Group II & 12 & 1401.00 & 4687.00 & 3525.33 & 899.25 \\
\hline Group IIA & 5 & 2891.00 & 4687.00 & 3749.40 & 692.48 \\
\hline Group IIB & 7 & 1401.00 & 4474.00 & 3365.29 & 1044.57 \\
\hline \multirow{5}{*}{$\begin{array}{l}\text { Statistical } \\
\text { significance }\end{array}$} & \multicolumn{3}{|c|}{ Group I, Group IIA and Group IIB (ANOVA) } & \multicolumn{2}{|c|}{$\mathrm{F}=1.342 ; \mathrm{p}=0.279$} \\
\hline & \multicolumn{3}{|c|}{ Between Group I and Group II (Student ' $t$ ' test) } & \multicolumn{2}{|c|}{$' t '=1.369 ; p=0.183$} \\
\hline & \multicolumn{3}{|c|}{ Between Group I and Group IIA (Student 't' test) } & \multicolumn{2}{|c|}{$' t '=0.508 ; p=0.617$} \\
\hline & \multicolumn{3}{|c|}{ Between Group I and Group IIB (Student ' $\mathrm{t}$ ' test) } & \multicolumn{2}{|c|}{$' t '=1.626 ; p=0.119$} \\
\hline & \multicolumn{3}{|c|}{ Between Group IIA and Group IIB (Student ' $t$ ' test) } & \multicolumn{2}{|c|}{$' t '=0.713 ; p=0.492$} \\
\hline
\end{tabular}

Table 6: Comparison of progesterone levels at different gestational age (32-35 weeks).

\begin{tabular}{|c|c|c|c|c|c|}
\hline & $\begin{array}{l}\text { Number of } \\
\text { subjects }\end{array}$ & $\begin{array}{l}\text { Minimum prg } \\
\text { level (pg/ml) }\end{array}$ & $\begin{array}{l}\text { Maximum prg } \\
\text { level (pg/ml) }\end{array}$ & $\begin{array}{l}\text { Mean prg level } \\
(\mathrm{pg} / \mathrm{ml})\end{array}$ & $\begin{array}{l}\text { Standard } \\
\text { deviation }\end{array}$ \\
\hline \multicolumn{6}{|c|}{ At gestational age 32-35 weeks } \\
\hline Group I & 30 & 2217.00 & 5656.00 & 3940.23 & 618.25 \\
\hline Group II & 24 & 2217.00 & 4832.00 & 3965.50 & 589.92 \\
\hline Group IIA & 23 & 2217.00 & 4832.00 & 3963.17 & 603.07 \\
\hline Group IIB & 1 & 4019.00 & 4019.00 & 4019.00 & - \\
\hline \multirow{5}{*}{$\begin{array}{l}\text { Statistical } \\
\text { significance }\end{array}$} & \multicolumn{3}{|c|}{ Group I, Group IIA and Group IIB (ANOVA) } & \multicolumn{2}{|c|}{$\mathrm{F}=0.015 ; \mathrm{p}=0.985$} \\
\hline & \multicolumn{3}{|c|}{ Between Group I and Group II (Student ' $t$ ' test) } & \multicolumn{2}{|c|}{$' \mathrm{t} '=0.152 ; \mathrm{p}=0.880$} \\
\hline & \multicolumn{3}{|c|}{ Between Group I and Group IIA (Student 't' test) } & \multicolumn{2}{|c|}{$\mathrm{t}^{\prime}=0.135 ; \mathrm{p}=0.893$} \\
\hline & \multicolumn{3}{|c|}{ Between Group I and Group IIB (Student ' $t$ ' test) } & \multicolumn{2}{|c|}{$\mathrm{t}^{\prime} \mathrm{\prime}=0.125 ; \mathrm{p}=0.901$} \\
\hline & \multicolumn{3}{|c|}{ Between Group IIA and Group IIB (Student ' $t$ ' test) } & \multicolumn{2}{|c|}{$' t '=0.091 ; p=0.929$} \\
\hline
\end{tabular}


At gestational age $<32$ weeks, neither intergroup difference in progesterone levels were found to be significant (Table 5).

At gestational age 32-35 weeks, intergroup difference in progesterone levels were not found to be significant (Table 6).
At gestational age $>35$ weeks $-<37$ weeks, group differences in progesterone levels were not found to be significant.

Group IIB as represented by only 7 subjects at gestational age 32 weeks and 1 subject each in 32-35 weeks and > $35-<37$ weeks (Table 7).

Table 7: Comparison of progesterone levels at different gestational age (> 35 - < 37 weeks).

\begin{tabular}{|c|c|c|c|c|c|}
\hline & $\begin{array}{l}\text { Number of } \\
\text { subjects }\end{array}$ & $\begin{array}{l}\text { Minimum prg } \\
\text { level (pg/ml) }\end{array}$ & $\begin{array}{l}\text { Maximum prg } \\
\text { level (pg/ml) }\end{array}$ & $\begin{array}{l}\text { Mean prg level } \\
(\mathrm{pg} / \mathrm{ml})\end{array}$ & $\begin{array}{l}\text { Standard } \\
\text { deviation }\end{array}$ \\
\hline \multicolumn{6}{|c|}{ At gestational age $>35$ weeks $-<37$ weeks } \\
\hline Group I & 4 & 3724.00 & 4651.00 & 4155.75 & 381.29 \\
\hline Group II & 14 & 2215.00 & 5129.00 & 3658.21 & 984.54 \\
\hline Group IIA & 13 & 2215.00 & 5129.00 & 3576.38 & 973.93 \\
\hline Group IIB & 1 & 4722.00 & 4722.00 & 4722.00 & - \\
\hline \multirow{5}{*}{$\begin{array}{l}\text { Statistical } \\
\text { significance }\end{array}$} & \multicolumn{3}{|c|}{ Group I, Group IIA and Group IIB (ANOVA) } & \multicolumn{2}{|c|}{$\mathrm{F}=1.262 ; \mathrm{p}=0.311$} \\
\hline & \multicolumn{3}{|c|}{ Between Group I and Group II (Student 't' test) } & \multicolumn{2}{|c|}{$' t '=0.972 ; p=0.345$} \\
\hline & \multicolumn{3}{|c|}{ Between Group I and Group IIA (Student ' $t$ ' test) } & \multicolumn{2}{|c|}{$\mathrm{t}^{\prime}{ }^{\prime}=1.142 ; \mathrm{p}=0.272$} \\
\hline & \multicolumn{3}{|c|}{ Between Group I and Group IIB (Student ' $t$ ' test) } & \multicolumn{2}{|c|}{$' t '=1.328 ; p=0.276$} \\
\hline & \multicolumn{3}{|c|}{ Between Group IIA and Group IIB (Student ' $t$ ' test) } & \multicolumn{2}{|c|}{$' t '=1.133 ; p=0.279$} \\
\hline
\end{tabular}

Table 8: Estriol levels (pg/ml) of study group and control group.

\begin{tabular}{|llllll|}
\hline & $\begin{array}{l}\text { Number of } \\
\text { subjects }\end{array}$ & $\begin{array}{l}\text { Minimum E3 } \\
\text { level }\end{array}$ & $\begin{array}{l}\text { Maximum E3 } \\
\text { level }\end{array}$ & Mean E3 level & $\begin{array}{c}\text { Standard } \\
\text { deviation }\end{array}$ \\
\hline Group I & 50 & 1048.00 & 4022.00 & 2691.72 & 681.08 \\
\hline Group IIA & 41 & 2227.00 & 4230.00 & 3512.85 & 586.16 \\
\hline Group IIB & 9 & 1259.00 & 3902.00 & 2513.78 & 947.55 \\
\hline \multirow{3}{*}{$\begin{array}{l}\text { Statistical } \\
\text { significance }\end{array}$} & Group I, Group IIA and Group IIB (ANOVA) & $\mathrm{F}=19.606 ; \mathrm{p}<0.001$ \\
\cline { 2 - 5 } & Between Group I and Group IIA (Student ' $\mathrm{t}$ ' test) & ' $\mathrm{t}$ ' $=6.088 ; \mathrm{p}<0.001$ \\
\cline { 2 - 5 } & Between Group I and Group IIB (Student ' $\mathrm{t}$ ' test) & ' $\mathrm{t}$ ' $=0.678 ; \mathrm{p}=0.500$ \\
\cline { 2 - 5 } & Between Group IIA and Group IIB (Student ' $\mathrm{t}$ ' test) & ' $\mathrm{t}$ ' $=4.111 ; \mathrm{p}<0.001$ \\
\hline
\end{tabular}

Table 9: Comparison of estriol levels $(\mathrm{pg} / \mathrm{ml})$ at different gestational age $(<32$ weeks).

\begin{tabular}{|c|c|c|c|c|c|}
\hline & $\begin{array}{l}\text { Number of } \\
\text { subjects }\end{array}$ & $\begin{array}{l}\text { Minimum E3 } \\
\text { level }\end{array}$ & $\begin{array}{l}\text { Maximum E3 } \\
\text { level }\end{array}$ & Mean E3 level & $\begin{array}{l}\text { Standard } \\
\text { deviation }\end{array}$ \\
\hline \multicolumn{6}{|c|}{ At gestational age $<32$ weeks } \\
\hline Group I & 16 & 1261.00 & 3716.00 & 2476.75 & 577.11 \\
\hline Group II & 12 & 1259.00 & 4096.00 & 2880.25 & 1056.04 \\
\hline Group IIA & 5 & 2618.00 & 4096.00 & 3631.60 & 597.58 \\
\hline Group IIB & 7 & 1259.00 & 3902.00 & 2343.57 & 1000.01 \\
\hline \multirow{5}{*}{$\begin{array}{l}\text { Statistical } \\
\text { significance }\end{array}$} & \multicolumn{3}{|c|}{ Group I, Group IIA and Group IIB (ANOVA) } & \multicolumn{2}{|c|}{$\mathrm{F}=5.991 ; \mathrm{p}=0.007$} \\
\hline & \multicolumn{3}{|c|}{ Between Group I and Group II (Student ' $t$ ' test) } & \multicolumn{2}{|c|}{$' \mathrm{t} '=1.297 ; \mathrm{p}<0.001$} \\
\hline & \multicolumn{3}{|c|}{ Between Group I and Group IIA (Student 't' test) } & \multicolumn{2}{|c|}{$' \mathrm{t} '=3.876 ; \mathrm{p}=0.001$} \\
\hline & \multicolumn{3}{|c|}{ Between Group I and Group IIB (Student ' $t$ ' test) } & \multicolumn{2}{|c|}{$' \mathrm{t} '=0.406 ; \mathrm{p}=0.119$} \\
\hline & \multicolumn{3}{|c|}{ Between Group IIA and Group IIB (Student ' $t$ ' test) } & \multicolumn{2}{|c|}{$' t '=2.552 ; p=0.029$} \\
\hline
\end{tabular}

Though estriol levels of Group II (3333.02 \pm 759.87 units) was found to be higher than that of Group I (2691.72 \pm 681.08 units) but this difference was not found to be statistically significant $(\mathrm{p}=0.222)$.
Estriol levels of Group IIA were found to be higher than that of Group I and Group IIB and this difference was significant $(\mathrm{p}<0.001)$. Estriol level of Group IIA was found to be significantly higher than that of Group llB. 
Though estriol levels of Group I was found to be higher than Group IIB but the difference was not significant (Table 8).

At gestational age $<32$ weeks, intergroup difference and all the between group difference in estriol levels except between Group I and Group IIB was found to be significant (Table 9).

At gestational age 32-35 weeks, intergroup difference between group differences in estriol levels except between Group I and Group IIB was found to be statistically significant (Table 10).

At gestational age >35 weeks - $<37$ weeks intergroup difference in estriol levels were not found to be statistically significant.

Group IIB was represented by only 7 subjects at gestational age 32 weeks and 1 each subject at 32-35 weeks and > 35 weeks - <37 weeks (Table 11).

Table 10: Comparison of estriol levels (pg/ml) at different gestational age (32-35 weeks).

\begin{tabular}{|c|c|c|c|c|c|}
\hline & $\begin{array}{l}\text { Number of } \\
\text { subjects }\end{array}$ & $\begin{array}{l}\text { Minimum E3 } \\
\text { level }\end{array}$ & $\begin{array}{l}\text { Maximum E3 } \\
\text { level }\end{array}$ & Mean E3 level & $\begin{array}{l}\text { Standard } \\
\text { deviation }\end{array}$ \\
\hline \multicolumn{6}{|c|}{ At gestational age $32-35$ weeks } \\
\hline Group I & 30 & 1048.00 & 4022.00 & 2727.73 & 712.66 \\
\hline Group II & 24 & 2533.00 & 4230.00 & 3658.63 & 512.24 \\
\hline Group IIA & 23 & 2533.00 & 4230.00 & 3698.48 & 484.22 \\
\hline Group IIB & 1 & 2742.00 & 2742.00 & 2742.00 & . \\
\hline \multirow{5}{*}{$\begin{array}{l}\text { Statistical } \\
\text { significance }\end{array}$} & \multicolumn{3}{|c|}{ Group I, Group IIA and Group IIB (ANOVA) } & \multicolumn{2}{|c|}{$\mathrm{F}=15.939 ; \mathrm{p}<0.001$} \\
\hline & \multicolumn{3}{|c|}{ Between Group I and Group II (Student 't' test) } & \multicolumn{2}{|c|}{$' \mathrm{t} '=5.379 ; \mathrm{p}<0.001$} \\
\hline & \multicolumn{3}{|c|}{ Between Group I and Group IIA (Student 't' test) } & \multicolumn{2}{|c|}{$' t '=5.609 ; p<0.001$} \\
\hline & \multicolumn{3}{|c|}{ Between Group I and Group IIB (Student ' $t$ ' test) } & \multicolumn{2}{|c|}{$' t '=0.020 ; p=0.984$} \\
\hline & \multicolumn{3}{|c|}{ Between Group IIA and Group IIB (Student ' $t$ ' test) } & \multicolumn{2}{|c|}{$' t '=1.934 ; p=0.066$} \\
\hline
\end{tabular}

Table 11: Comparison of estriol levels (pg/ml) at different gestational age ( $>35$ - < 37 weeks).

\begin{tabular}{|c|c|c|c|c|c|}
\hline & $\begin{array}{l}\text { Number of } \\
\text { subjects }\end{array}$ & $\begin{array}{l}\text { Minimum E3 } \\
\text { level }\end{array}$ & $\begin{array}{l}\text { Maximum E3 } \\
\text { level }\end{array}$ & Mean E3 level & $\begin{array}{l}\text { Standard } \\
\text { deviation }\end{array}$ \\
\hline \multicolumn{6}{|c|}{ At gestational age $>35$ weeks $-<37$ weeks } \\
\hline Group I & 4 & 2659.00 & 3723.00 & 3281.50 & 527.72 \\
\hline Group II & 14 & 2227.00 & 4128.00 & 3162.93 & 593.61 \\
\hline Group IIA & 13 & 2227.00 & 4128.00 & 3138.77 & 610.64 \\
\hline Group IIB & 1 & 3477.00 & 3477.00 & 3477.00 & . \\
\hline \multirow{5}{*}{$\begin{array}{l}\text { Statistical } \\
\text { significance }\end{array}$} & \multicolumn{3}{|c|}{ Group I, Group IIA and Group IIB (ANOVA) } & \multicolumn{2}{|c|}{$\mathrm{F}=0.212 ; \mathrm{p}=0.811$} \\
\hline & \multicolumn{3}{|c|}{ Between Group I and Group II (Student ' $t$ ' test) } & \multicolumn{2}{|c|}{$' t '=0.359 ; p=0.724$} \\
\hline & \multicolumn{3}{|c|}{ Between Group I and Group IIA (Student ' $t$ ' test) } & \multicolumn{2}{|c|}{$' t '=0.420 ; p=0.682$} \\
\hline & \multicolumn{3}{|c|}{ Between Group I and Group IIB (Student ' $t$ ' test) } & \multicolumn{2}{|c|}{${ }^{\prime} \mathrm{t} '=0.331 ; \mathrm{p}=0.762$} \\
\hline & \multicolumn{3}{|c|}{ Between Group IIA and Group IIB (Student ' $t$ ' test) } & \multicolumn{2}{|c|}{$' t '=0.534 ; p=0.603$} \\
\hline
\end{tabular}

E3 was evaluated for prediction of preterm delivery at a cut-off with a larger value indicating positive by ROC curve. Area under curve findings were 0.815 (indicating a projected accuracy of $81.5 \%$ ) for E3. On evaluating E3, a cut-off value $>3107.50$ was predicted to be $75.6 \%$ sensitive and $72.9 \%$ specific. Efficacy of Prg was evaluated for a smaller value indicating a higher chance of preterm delivery, the ROC analysis showed area under curve to be 0.526 which did not show a significant association with the outcome $(p=0.656)$, hence no further attempt to find out a suitable cut-off value was made for.

\section{DISCUSSION}

Various biochemical parameters proposed have high potential to identify those symptomatic and asymptomatic patients who are at greatest risk for preterm birth and who subsequently may require more intensive observation and tocolysis and therapy for fetal lungs maturation. Conversely if low risk of preterm birth is diagnosed the need for aggressive tocolysis, prolonged hospitalization and closed follow up visits may be reduced. Till date several biochemical markers have been studied but none have proved to be a gold standard. 
In this study we observed that saliva progesterone levels of pregnant women with preterm delivery (study group IIA) was lower than the control group (Group l) but the difference was statistically not significant (3814.46 $\pm 751.14 \mathrm{pg} / \mathrm{ml}$ versus $3945.16 \pm 577.11 \mathrm{pg} / \mathrm{ml}, \mathrm{p}$ $=0.351)$.

In study group IIB (patients who came with preterm labour pain and delivered at terms) the mean level of progesterone was $3588.67 \pm 1022-60 \mathrm{pg} / \mathrm{ml}$. This value is lower than the mean value of control group $(3945.16 \pm 577.11 \mathrm{pg} / \mathrm{ml})$ but difference was not statistically significant $(\mathrm{p}>0.140)$.

Similar to our study, Lachelin et al, reported that saliva progesterone concentrations in women who delivered before 34 weeks following the spontaneous onset of preterm labour were significantly lower than those of the terms group $(\mathrm{p}=0.009)$ or those delivering after spontaneous onset between 34 and 37 weeks of gestation $(\mathrm{p}=0.007){ }^{9}$

Klebanoff et al in their study on 386 women made similar observation and reported low progesterone concentration associated with increased risk of preterm labour. ${ }^{10}$

In the present study the mean estriol level in control group was $2691.72 \pm 681.08 \mathrm{pg} / \mathrm{ml}$ and in study Group II was $3333.02 \pm 759.87 \mathrm{pg} / \mathrm{ml}$, which was higher than control group but the difference was not statistically significant $(\mathrm{p}=0.222)$.

Mean value of study Group IIA $(3512.85 \pm 586.16 \mathrm{pg} / \mathrm{ml})$ was higher as compared to the mean value of control group $(2691.72 \pm 681.08 \mathrm{pg} / \mathrm{ml})$ and difference was statistically significant ( $\mathrm{p}$ value $<0.001$ ). Similar observation was made by McGregor et al and they reported that detection of an early estriol surge or increased level (> $2.3 \mathrm{ng} / \mathrm{ml}$ ) may be clinically helpful in identifying women at elevated risk for preterm labour. ${ }^{11}$

In our study the mean of saliva estriol in study Group II in gestational age < 32 weeks was $2880.25 \pm 1056.24$ $\mathrm{pg} / \mathrm{ml}$ which was significantly higher than mean value of estriol in control Group I $(2476.75 \pm 577.11 \mathrm{pg} / \mathrm{ml}, \mathrm{p}<$ $0.001)$. The mean value of saliva estriol in Group IIA $(3512.85 \pm 586.16 \mathrm{pg} / \mathrm{ml})$ was significantly raised than control Group I ( $\mathrm{p}<0.001)$. On comparing the mean value of Group I (control group) and Group IIB $(2343.57 \pm 1000.01 \mathrm{pg} / \mathrm{ml})$ no statistically significant difference was seen.

The mean value of estriol in gestational age between $>35$ weeks and $<37$ weeks was not significantly different in both study group and control group. Lachelin et al, studied saliva estriol in these gestational age group $<34$ weeks, 34-37 weeks and > 37 weeks but they found that there was no significant difference in between different gestational age group. ${ }^{9}$
We were unable to find any cut off value for saliva progesterone. In this study the cut off value for E3 was $\geq$ $3107.50 \mathrm{pg} / \mathrm{ml}(3.1 \mathrm{ng} / \mathrm{ml})$ with sensitivity and specificity of $75.6 \%$ and $72.9 \%$ respectively for women liable to undergo preterm labor. So further studies with larger sample are required to evaluate the accuracy of the saliva estriol for identifying those symptomatic and who are at risk for preterm birth.

\section{CONCLUSION}

Thus, there is significant rise in the level of saliva estriol, it can be used as a predictor for detecting symptomatic and asymptomatic women at risk for preterm birth. Whole saliva collected in non - invasive manner by individual with modest training including patient. However, before saliva estriol is used as diagnostic test, further investigation are necessary to evaluate its accuracy as a biochemical marker for predicting preterm delivery.

\section{Funding: No funding sources Conflict of interest: None declared \\ Ethical approval: The study was approved by the Institutional Ethics Committee}

\section{REFERENCES}

1. Lumley J. Defining the problem the epidemiology of preterm birth. BJOG. 2003;110Suppl 20:3-7.

2. Creasy RK, Merkatz IR. Prevention of preterm birth: Clinical opinion. Obstet Gynecol. 1990;76(1 Suppl):2S-4S.

3. McGarrigle HHG, Lachelin GCL. Increasing saliva (free) estriol to progesterone ratio in late pregnancy: a role for estriol in intiating spontaneous onset of labour at term. BMJ. 1984;287:457-9.

4. Darne J, McGarrigle HHG, Lachelin GCL. Saliva estriol, estadiol, estrone and progesterone levels in pregnancy: spontaneous labour at term is preceded by arise in the saliva estrol progesterone ratio. BJOG. 1987;94:227-35.

5. Kundu N, Novak N, Petersen LP. Salivary unconjugated estriol levels in normal third trimester pregnancy-direct correlation with serum levels. Steroids. 1983;41:145-53.

6. Vining RF, McGinley R, Rice BV. Saliva estriol measurements: an alternative to the assay of serum unconjugated estriol in assessing feto-placental function. J Clin Endocrinol Metab. 1983b;56:454-60.

7. Luisi M, Franchi F, Kicovic PM, Silvestri D, Cossu G, Catarsi AL, et al. Radioimmunoassay for progesterone in human saliva during the menstrual cycle. J Steroid Biochem. 1981;14:1069-73.

8. Choe JK, Khan-Dawood FS, Dawood MY. Progesterone and estradiol in the saliva and plasma during the menstrual cycle. Am J Obstet Gynecol 1983;147:557-62.

9. Lachelin GCL, McGarrigle, Seed PL. Low saliva progesterone concentrations are associated with 
spontaneous every preterm labour (before 34 weeks of gestation) in women at increased risk of preterm delivery. Br J Obstet Gynaecol. 2009;116:1515-9.

10. Klebanoff MA, Meis PJ, Dombrowski MP, Zhao Y, Moawad AH, Northen A, et al. Salivary progesterone and estriol among pregnant women treated with 17a- hydroxyprogesterone caproate or placebo. Am J Obstet Gynecol. 2008;199:506.e1-506.e7.

11. McGregor JA, Jackson GM, Lacheln GCL, Goodwin TM, Artal R, Hastings C, et al. Salivary estriol as risk assessment for preterm labour: a prospective trial. Am J Obstet Gynecol. 1995;173:1337-42.

Cite this article as: Yadav R, Singh U. Study of changes in levels of salivary estriol and progesterone in preterm labor in comparison to normal pregnancy. Int J Reprod Contracept Obstet Gynecol 2019;8:4660-7. 